\title{
Adding New Perspectives to the Zero-Price Effect: The Role of Non-monetary Cost Perceptions
}

\author{
Björn A. Hüttel, Christian J. Wagner, and Jan H. Schumann
}

\begin{abstract}
Research shows that free offers lead to positive affective consumer reactions which result in an irrationally high demand (i.e., zero-price effect). Based on the assumption of constant costs prior research argues that consumers attribute extra benefits to free offers. Yet, so far there is no empirical evidence for this claim. In this research we test this assumption but also challenge the assumption of constant costs. In a first study in the context of free e-services we show that consumers perceive not only the benefits of free offers but also their non-monetary costs. Moreover, we show that consumers actually overemphasize the benefits of a free e-service but also less perceive its non-monetary costs (i.e., advertising intrusiveness). Further we show that the lower perception of non-monetary costs does not occur for critical nonmonetary costs like personal data. In a second study we show that both the attribution of extra benefits and the lower perception of non-monetary costs disappear when the offer is mainly utilitarian. Consequently under these conditions also the zero-price effect vanishes. This research extends prior marketing theory by adding a new explanatory aspect to and outlining boundary conditions of the zero-price effect. The results are also relevant for marketing practitioners currently offering free services or products or considering switching to a free business model.
\end{abstract}

B.A. Hüttel $(\bowtie) \bullet$ C.J. Wagner $\bullet$ J.H. Schumann

University of Passau, Passau, Germany

e-mail: Bjoern.Huettel@uni-passau.de; Christian.Wagner@uni-passau.de; Jan.Schumann@

uni-passau.de 